\title{
Optimization of femtosecond laser cutting of a biodegradable polymer for medical devices manufacturing
}

\author{
Bogusz Stępak, ${ }^{* 1}$ Arkadiusz J. Antończak, ${ }^{2}$ and Krzysztof M. Abramski ${ }^{2}$ \\ ${ }^{I}$ Faculty of Microsystem Electronics and Photonics, ${ }^{2}$ Faculty of Electronics, \\ Wrocław University of Science and Technology, Wybrzeże Wyspiańskiego 27, 50-370 Wrocław
}

Received November 17, 2016; accepted December 19, 2016; published December 31, 2016

\begin{abstract}
This paper describes the experimental parameters involved in the femtosecond laser micromachining of biodegradable poly(L-lactide) which is frequently used in biomedical applications such as vascular stents or scaffolds. We investigated the influence of laser pulse energy, scanning strategy and number of overscans on the laser cutting throughput. The process parameters that enable reducing a heat affected zone were determined. As a result, the optimal scanning strategy was determined in order to obtain high aspect ratio trenches in a $380 \mu \mathrm{m}$ thick biodegradable polymer sheet.
\end{abstract}

Over the last decade we have observed the increasing involvement of femtosecond lasers for manufacturing biomedical devices made of biodegradable polymers. A small beam diameter and short pulse duration enable forming complex, submillimeter geometrical shapes in polymers such as vascular stents which cannot be manufactured using traditional techniques e.g. injection moulding or mechanical processing. The shortening of pulse duration below electron cooling time enables reduction of thermal and mechanical damage to the surrounding material. Furthermore, extremely high power density causes multiphoton absorption that allows for processing wide-bandgap dielectrics. Together with high pulse energy in the range of tens of microjoules attainable in modern industrial constructions, femtosecond lasers appear as a perfect tool for efficient machining of polymers.

There are, however, several limitations regarding the use of femtosecond lasers for processing heat sensitive biodegradable polymers. As it was reported previously, laser micromachining with ultrashort pulse lasers can result in thermal load affecting the surrounding material causing its melting, crack formation [1-2] or crystallisation [3]. Nevertheless, heat accumulation appears mainly for a high repetition rate, high pulse energy and multiple scanning cycles. Depending on the relation between laser wavelength, fluence, repetition frequency and pulse overlapping, the process can be designed to be a-thermal [2]. In order to preserve a relatively high process throughput one can apply different strategies such as decreasing pulse overlapping [4] or

*E-mail: bogusz.stepak@ @wr.edu.pl using gas assist [2]. Another issue affecting the processing speed is the reflection and scattering of incident light in a $\mathrm{V}$-shaped groove causing a decrease of the ablation rate with an increasing number of over-scans [5].

This article presents the results of the cutting process optimisation of medical grade poly(L-lactide) (PLLA RESOMER $_{\circledast}$ L $210 \mathrm{~S}$, Evonik Biomaterials). PLLA is a semicrystalline aliphatic polyester which is transparent in a wide optical window beginning from $250 \mathrm{~nm}$ up to near infrared. The thickness of an amorphous polymer sheet prepared using a hydraulic press was $380 \pm 10 \mu \mathrm{m}$. The experiments were performed using $\mathrm{s}$ laser from Active Fiber Systems Jena with the pulse duration $\tau=450 \mathrm{fs}$, wavelength $\lambda=515 \mathrm{~nm}$ and fixed pulse repetition frequency $10 \mathrm{kHz}$. The focal length of the lens was $163 \mathrm{~mm}$ and the beam diameter in the focal plane was $25 \mu \mathrm{m}$.

The laser cutting trials were performed in ambient air using a test pattern that consists of six grooves with a length of $1 \mathrm{~mm}$ and a different width (Fig. 1). The grooves were produced by bidirectional scanning in the $y$ direction each with the hatching distance $10 \mu \mathrm{m}$.

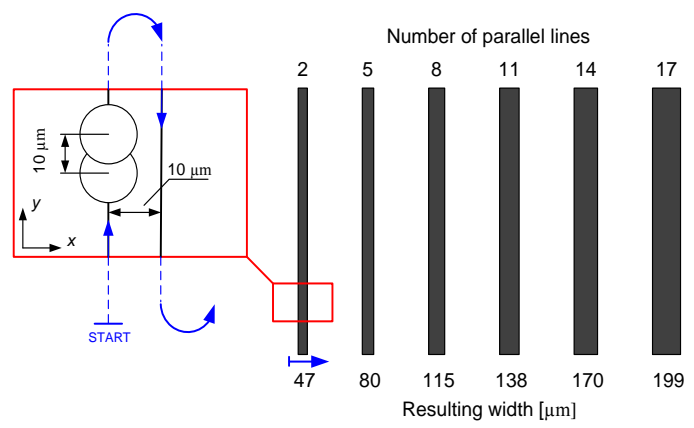

Fig. 1. Test pattern and scanning routine.

The scanning speed of $100 \mathrm{~mm} / \mathrm{s}$ resulted in $10 \mu \mathrm{m}$ pulse spacing along the scan direction. In consequence, the $x y$ pulse separation was $10 \mu \mathrm{m}$ during all experiments. A different width of grooves was obtained by increasing the number of parallel lines from 2 up to 17 .

The width was measured at the top surface. The test pattern was performed using a different laser fluence $1.4 \div 5.2 \mathrm{~J} / \mathrm{cm}^{2}$ and a number of overscans $10 \div 60$. 

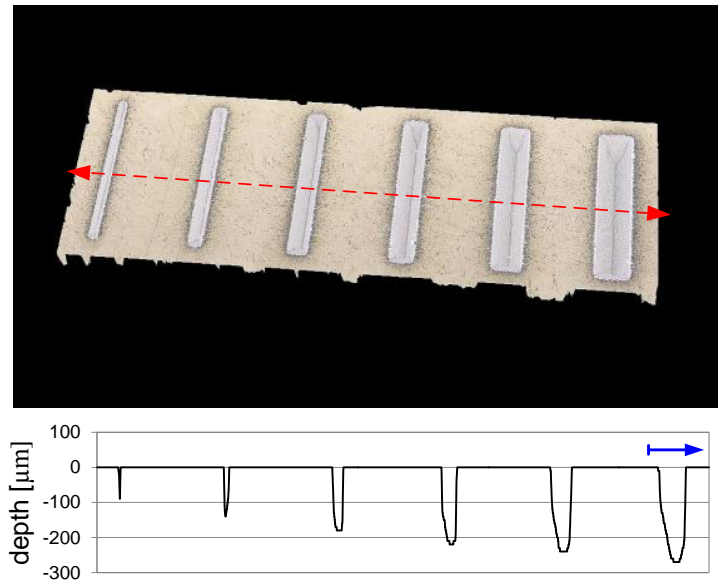

Fig. 2. The 3D image and profile of test pattern in PLLA obtained for 50 overscans and a fluence of $2.1 \mathrm{~J} / \mathrm{cm}^{2}$

The profile of grooves presented in Fig. 2 shows that there is strong dependence between the depth and number of parallel lines used, thus the kerf width. The asymmetric profile of a single groove is caused by the direction of scanning from the left side to the right. In order to find optimal parameters for a cutting process it is necessary to investigate the relation between the number of lines and the number of overscans in relation to the applied laser fluence.

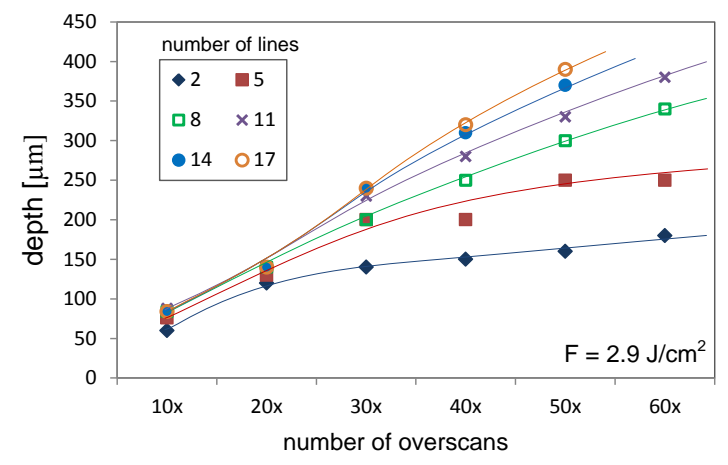

Fig. 3. The measured depth of grooves as a function of the number of overscans for a different number of parallel lines at a fluence of $2.9 \mathrm{~J} / \mathrm{cm}^{2}$.

As can be observed in Fig. 3, the depth of grooves/ablation rate saturates with an increasing overscan number for 2 and 5 lines which correspond to 47 and $80 \mu \mathrm{m}$ kerf width, respectively. The increase of groove width up to $115 \mu \mathrm{m}$ ( 8 lines) results in noticeable increase of the ablation rate in a range of $40 \div 60$ overscans. An interesting effect can be seen for 10 and 20 repetitions where the depth is almost independent of the groove width while for 30 repetitions the number of lines plays an important role. Figure 4 presents data points from the Fig. 3 in relation to the resulting speed of cutting which was calculated as the speed of the scanner $100 \mathrm{~mm} / \mathrm{s}$ divided by the product of the number of lines and number of overscans. This representation allows one to shorten the processing time and have the desired kerf width. As can be noted, there are limitations regarding the maximum depth which can be achieved using 2, 5 and 8 lines using up to 60 overscans. Nevertheless, the highest cutting speed for the material with a thickness up to $340 \mu \mathrm{m}$ can be achieved using $2 \div 8$ parallel lines. The use of 14,17 lines and 60 overscans allowed us to cut through a $380 \mu \mathrm{m}$ polymer sheet. It is worth emphasizing that the taper angle between the cut walls is not considered here.

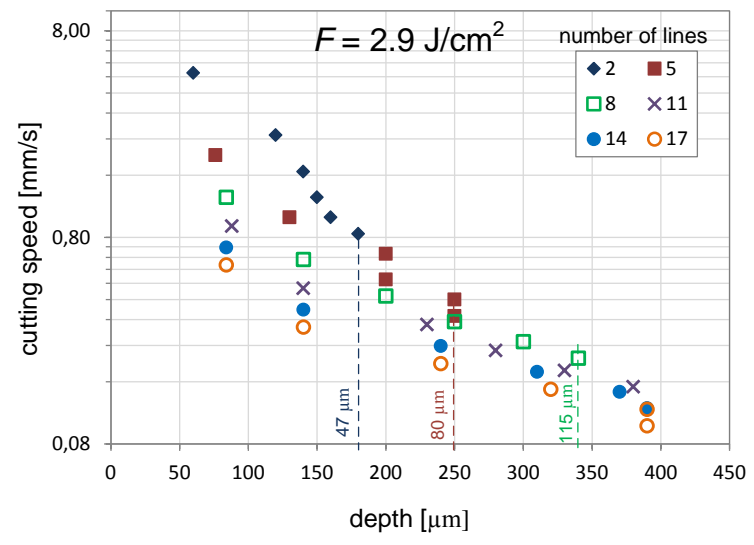

Fig. 4. The cutting speed for different sets of parameters with an indicated kerf width in relation to the obtained depth.

The cutting speed can be increased by changing the laser fluence, repetition frequency or pulse overlapping. However, a-thermal character of machining has to be preserved. Cutting trials were performed also for different laser fluence values. As shown in Fig. 5, the increase of fluence up to $4.7 \div 5.2 \mathrm{~J} / \mathrm{cm}^{2}$ enabled cutting through a PLLA sheet using 30 overscans.

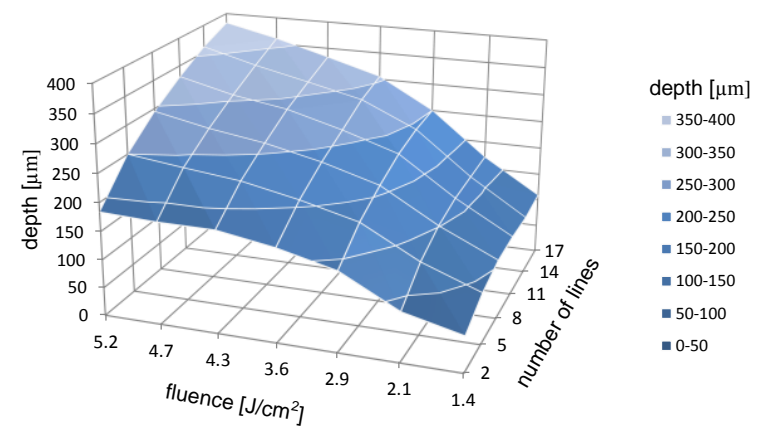

Fig. 5. The depth of grooves as a function of laser fluence and the number of lines for 30 overscans.

Ablation rate enhancement is most visible in the case of a higher number of lines $11 \div 17$. For narrow grooves $2 \div 8$ the ablation rate saturates faster with increasing power. The cutting speed at the laser fluence $F=5.2 \mathrm{~J} / \mathrm{cm}^{2}$ calculated for different scanning strategies is shown in 
Fig. 6. An improvement is noticeable in comparison to the maximum process speed obtained with $F=2.9 \mathrm{~J} / \mathrm{cm}^{2}$. There is, however, a limiting factor for increasing pulse energyheat affected zone (HAZ).

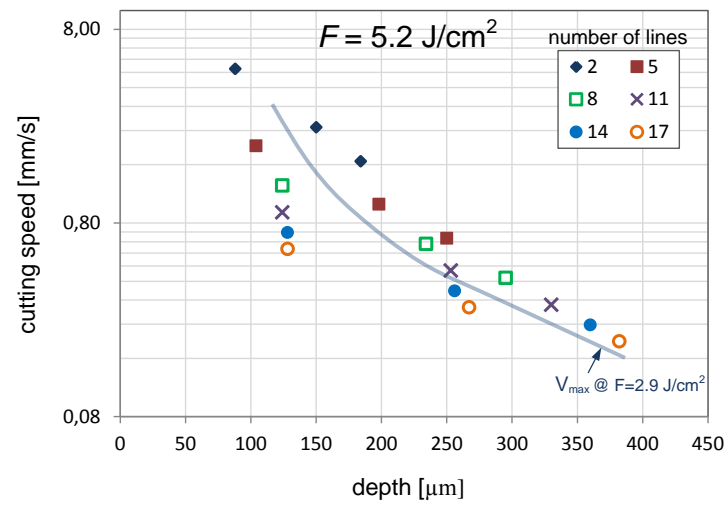

Fig. 6. The cutting speed as a function of the depth at a fluence of $5.2 \mathrm{~J} / \mathrm{cm}^{2}$ and marked maximum cutting speed obtained using $2.9 \mathrm{~J} / \mathrm{cm}^{2}$.

We observed that for high fluence and a high number of parallel lines there is an HAZ surrounding the grooves. Nonetheless, it is worth noting that such an effect can be reduced by increasing the length of a groove which would avoid heat accumulation. The HAZ formation was not observed for a number of lines $2 \div 5$ at any of used process parameters. The process parameters enabling the highest cutting speed without HAZ formation for certain material thickness are collected in Table 1.

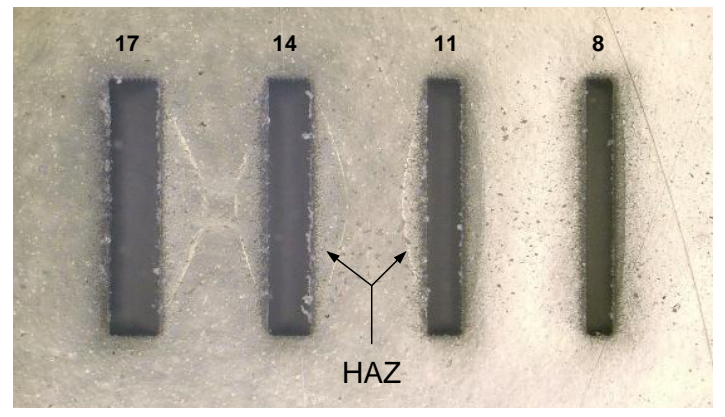

Fig. 7. Heat affected zone observed for 20 overscans and a fluence of $5.2 \mathrm{~J} / \mathrm{cm}^{2}$ for an indicated number of lines.

A similar HAZ in PLLA connected with heat accumulation and caused by $\mathrm{CO}_{2}$ or $\mathrm{ArF}$ excimer laser cutting was observed and characterized previously [6-7]. The HAZ visible in Fig. 7 is a result of the increase in material temperature above glass transition temperature $\left(\sim 65^{\circ} \mathrm{C}\right.$ for PLLA) during the process.

As presented in Fig. 8, the cutting of a polymer sheet thicker than $300 \mu \mathrm{m}$ can be considered as inefficient. The processing speed decreases exponentially while the kerf width increases reaching the values which are limiting precision.
Table 1. Optimal laser machining parameters for HAZ-free cutting of desired PLLA thickness

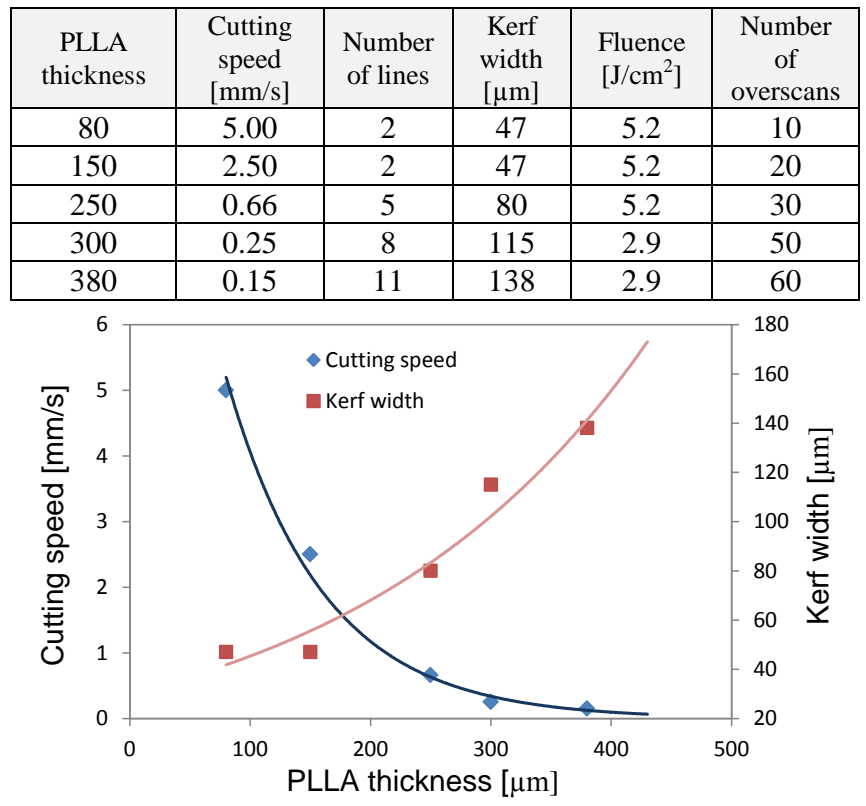

Fig. 8. The relation between maximum process speed and kerf width for certain PLLA thickness

In comparison to other reports on femtosecond laser cutting of poly(L-lactide) with a single-scan procedure [2], the obtained process speed without gas assist is at least one order of magnitude higher. Despite the femtosecond pulse duration, there is still a need to optimize the process in order to avoid residual heat accumulation especially in the case of heat sensitive medical grade polymers.

This research was conducted in cooperation with the Fraunhofer Institute Material and Beam Technology IWS in Dresden, Germany. The author received financial support to prepare a Ph.D. thesis from the Polish National Science Centre in the frame of doctoral scholarship decision number: DEC-2015/16/T/ST8/00483. The research was supported by statutory funds from the Dept. of Field Theory, Electronics Systems and Optoelectronics, Wroclaw University of Technology under grant No. 0401/0094/16.

\section{References}

[1] W. Jia et al., Opt. Expr. 21, 23 (2015).

[2] F. Hendricks, R. Patel, V.V. Matylistsky, Proc. SPIE 9355, 935502 (2015).

[3] W.Y. Yeong et al., Adv. Eng. Mater. 4, 12 (2010).

[4] K. Stolberg et al., Proc. SPIE 8968, 89680E (2014).

[5] F. Hendricks et al., Appl. Phys. A 117, 1 (2014).

[6] A. Antończak et al., Polym. Deg. Stab. 109, 97 (2014)

[7] B. Stępak et al., Proc SPIE 9736, $97361 T$ (2016). 